\title{
Antiviral efficacy of short-hairpin RNAs and artificial microRNAs targeting foot-and-mouth disease virus
}

\author{
Anabella Currá ${ }^{1}$, Marco Cacciabue ${ }^{1}$, Maria Jose Gravisaco ${ }^{1}$, Sebastian Asurmendi ${ }^{1}$, Oscar Taboga ${ }^{\text {Corresp., } 1}{ }^{1}$, Maria I \\ Gismondi ${ }^{\text {Corresp. } 1}$ \\ 1 Instituto de Agrobiotecnología y Biología Molecular (IABiMo), Instituto Nacional de Tecnología Agropecuaria (INTA), Consejo Nacional de Investigaciones \\ Científicas y Técnicas (CONICET), Hurlingham, Buenos Aires, Argentina \\ Corresponding Authors: Oscar Taboga, Maria I Gismondi \\ Email address: taboga.oscaralberto@inta.gob.ar, gismondi.maria@inta.gob.ar
}

RNA interference (RNAi) is a well-conserved mechanism in eukaryotic cells that directs posttranscriptional gene silencing through small RNA molecules. RNAi has been proposed as an alternative approach for rapid and specific control of viruses including foot-andmouth disease virus (FMDV), the causative agent of a devastating animal disease with high economic impact. The aim of this work was to assess the antiviral activity of different small RNA shuttles targeting the FMDV RNA-dependent RNA polymerase coding sequence (3D). Three target sequences were predicted within 3D considering RNA accessibility as a major criterion. The silencing efficacy of short-hairpin RNAs (shRNAs) and artificial microRNAs (amiRNAs) targeting the selected sequences was confirmed in fluorescent reporter assays. Furthermore, BHK-21 cells transiently expressing shRNAs or amiRNAs proved 70 to $>95 \%$ inhibition of FMDV growth. Interestingly, dual expression of amiRNAs did not improve FMDV silencing. Lastly, stable cell lines constitutively expressing amiRNAs were established and characterized in terms of antiviral activity against FMDV. As expected, viral replication in these cell lines was delayed. These results show that the target RNAaccessibility-guided approach for RNAi design rendered efficient amiRNAs that constrain FMDV replication. The application of amiRNAs to complement FMDV vaccination in specific epidemiological scenarios shall be explored further. 


\section{Antiviral efficacy of short-hairpin RNAs and artificial \\ 2 microRNAs targeting foot-and-mouth disease virus}

3 Anabella Currá ${ }^{1}$, Marco Cacciabue ${ }^{1}$, María José Gravisaco ${ }^{1}$, Sebastián Asurmendi ${ }^{1}$, Oscar

4 Taboga $^{1}$, María Inés Gismondi ${ }^{1}$

$5{ }^{1}$ Instituto de Agrobiotecnología y Biología Molecular (IABIMO), Instituto Nacional de Tecnología

6 Agropecuaria (INTA), Consejo Nacional de Investigaciones Científicas y Técnicas (CONICET),

7 Hurlingham, Buenos Aires, Argentina

9 Corresponding Authors:

10 Oscar Taboga ${ }^{1}$

11 De los Reseros y N. Repetto s/n, Hurlingham, Buenos Aires, 1686, Argentina.

12 Email address: taboga.oscaralberto@inta.gob.ar

María Inés Gismondi ${ }^{1}$

De los Reseros y N. Repetto s/n, Hurlingham, Buenos Aires, 1686, Argentina. 


\section{Abstract}

22

23

24

RNA interference (RNAi) is a well-conserved mechanism in eukaryotic cells that directs posttranscriptional gene silencing through small RNA molecules. RNAi has been proposed as an alternative approach for rapid and specific control of viruses including foot-and-mouth disease virus (FMDV), the causative agent of a devastating animal disease with high economic impact. The aim of this work was to assess the antiviral activity of different small RNA shuttles targeting the FMDV RNA-dependent RNA polymerase coding sequence (3D). Three target sequences were predicted within 3D considering RNA accessibility as a major criterion. The silencing efficacy of short-hairpin RNAs (shRNAs) and artificial microRNAs (amiRNAs) targeting the selected sequences was confirmed in fluorescent reporter assays. Furthermore, BHK-21 cells transiently expressing shRNAs or amiRNAs proved 70 to $>95 \%$ inhibition of FMDV growth. Interestingly, dual expression of amiRNAs did not improve FMDV silencing. Lastly, stable cell lines constitutively expressing amiRNAs were established and characterized in terms of antiviral activity against FMDV. As expected, viral replication in these cell lines was delayed. These results show that the target RNA-accessibility-guided approach for RNAi design rendered efficient amiRNAs that constrain FMDV replication. The application of amiRNAs to complement FMDV vaccination in specific epidemiological scenarios shall be explored further.

Keywords

FMDV, antivirals, RNA interference, target accessibility, RNA structure, transgenic cell culture 


\section{1. Introduction}

43 Foot-and-mouth disease virus (FMDV) is the causative agent of a highly devastating disease of

44 cloven-hoofed animals leading to enormous economic losses. FMDV belongs to the Aphthovirus

45 genus in the Picornaviridae family. There are 7 FMDV serotypes (A, O, C, Asia1, SAT1, SAT2,

46 SAT3) and several subtypes.

47 The viral particle is $\sim 25-30 \mathrm{~nm}$ in diameter and consists of a single stranded $(+)$ RNA molecule

48 surrounded by an icosahedric capsid composed by the four structural viral proteins VP1 to VP4.

49 Viral infection begins with the interaction of the FMDV capsid with cellular receptors $\left(\alpha_{V}\right.$

50 integrins in animal hosts, additionally heparansulfate and a newly identified receptor in cultured

51 cells) (Dicara et al., 2008; Lawrence et al., 2016). Receptor-mediated endocytosis guides the

52 viral particles to the cytoplasm, where the whole replication cycle takes place. Acidification of

53 endosomes drives disassembly of the viral capsid and release of the FMDV genome into the

54 cytoplasm, where it is immediately translated in a cap-independent manner to produce the viral

55 polyprotein. Co- and post-translational processing of the FMDV polyprotein originates viral

56 protein precursors and ultimately the mature structural and non-structural proteins (Grubman \&

57 Baxt, 2004). Finally, capsids are assembled and newly synthesized genomes are encapsidated

58 and released from the cell in a lytic way. The replication cycle is fast, taking approximately 6-8

59 hours in cell culture.

60 During replication, viral RNA molecules are synthesized by the viral RNA-dependent RNA

61 polymerase (RdRp) 3Dpol. As other viral RdRps, the enzyme lacks proofreading activity, which

62 contributes to the generation of mutated FMDV genomes in every replication cycle. Hence, in an

63 infected host (cell or animal) the virus appears as a swarm of different but closely related viral 
64 variants which are called the viral quasispecies (Domingo et al., 2003; Domingo, Sheldon \&

65 Perales, 2012; Andino \& Domingo, 2015). The quasispecies nature of FMDV facilitates its rapid

66 adaptation to unfavorable environments.

67 Following current standards of the World Organisation for Animal Health (OIE), countries are 68 divided according to their foot-and-mouth disease (FMD) status in FMD-free countries with or without vaccination (almost all Americas, Europe and Oceania) and FMD-endemic countries (parts of Africa and Asia). Current approved FMD vaccines are inactivated viruses formulated with oil-based adjuvants. Although they are effective, inactivated vaccines show a number of disadvantages including lack of cross-protection between serotypes and even subtypes, inability to induce long-lasting immunity (there is a need of re-vaccination to maintain protection) and risk of incomplete inactivation during manufacturing. Moreover, current vaccines take $\sim 7$ days to induce protection (Golde et al., 2005). Thus, there is a need of developing new tools against FMDV that may complement vaccination and reduce the risk of infection especially during 77 outbreaks. RNA interference (RNAi) is a well-conserved mechanism in eukaryotic cells that directs posttranscriptional gene silencing (Fischer, 2015). RNAi is mediated through small RNA molecules that guide ribonucleprotein complexes to their cognate mRNAs and lead to their degradation and/or inhibition of translation. There are many types of small RNAs, including microRNAs (miRNAs) and short interfering RNAs (siRNAs). The former are endogenous molecules encoded in the cellular genome that regulate gene expression. In animals, biogenesis of miRNAs involves RNA polymerase II-mediated transcription of a primary miRNA (primiRNA), which is further processed by the RNAse III enzyme Drosha to pre-miRNA hairpins. 
87 miRNAs that exert their function in the cytoplasm of the cell. In turn, siRNAs are exogenous

88 molecules that may be introduced into the cell either as mature siRNAs or as DNA precursors by

89

transfection or by virus-mediated transduction (Castanotto \& Rossi, 2009). Importantly, the degree of complementarity between the small RNA and the target RNA defines the mechanism of posttranscriptional gene silencing: while perfect base complementarity mainly drives target RNA degradation by endonucleolytic cleavage, partial complementarity can lead to translational repression. In animals, the canonical siRNA pathway involves perfect siRNA:target RNA recognition, whereas binding of most miRNAs demands base pairing of the 'seed' region (mostly located at nucleotides 2-8 of the mature miRNA), allowing for imperfect target RNA recognition (reviewed in Carthew \& Sontheimer, 2009).

The use of RNAi as an antiviral tool has been explored against different viruses (reviewed in Qureshi et al., 2018 and Shah \& Schaffer, 2011). Indeed, RNAi mediators such as doublestranded or single-stranded siRNAs and plasmid or virus-encoded short hairpin RNAs (shRNAs) have been evaluated in different experimental settings against animal viruses including FMDV (Chen et al., 2004). Genetically modified animals expressing shRNAs targeting FMDV have also been developed with promising results (Jiao et al., 2013; Hu et al., 2015; Deng et al., 2017). Of note, shRNAs are potent RNAi mediators that usually are expressed at very high levels from RNA-polymerase III promoters; however, this can potentially lead to saturation of the cellular RNAi processing machinery. To overcome this drawback, artificial miRNAs (i.e. miRNAs rationally designed to target defined sequences) have been favored as RNAi shuttles since they exhibit increased safety with comparable efficacy (Boudreau, Martins \& Davidson, 2009). The efficacy of artificial miRNAs (amiRNAs) against FMDV has been investigated to a lesser extent (Du et al., 2011; Gismondi et al., 2014; Basagoudanavar et al., 2019). In this work, we further 
110 assess the efficacy of amiRNAs against FMDV and shRNAs targeting the same viral regions in

111 susceptible cells.

112

113

\section{Material and methods}

114

2.1 Cells and viruses

115

Baby hamster kidney (BHK-21 clone 13, ATCC CCL10) cells were obtained from the American Type Culture Collection and maintained in Dulbecco's modified Eagle's medium (DMEM, Life

117 Technologies, Grand Island, NY, USA) supplemented with 10\% fetal bovine serum (FBS) and antibiotics (Gibco-BRL/Invitrogen, Carlsbad, CA, USA) at $37^{\circ} \mathrm{C}$ with $5 \% \mathrm{CO} 2$. Stably transformed cell lines were grown as described in (Gismondi et al., 2014) .

FMDV A/Arg/01 isolate A01L (GenBank accession number KY404934) was obtained from the

121 National Institute for Animal Health (SENASA, Argentina). All experiments were conducted using fourth cell passages in BHK-21 cells. Experiments involving active FMDV were performed in the BSL-4 OIE facility at the IVIT (INTA-CONICET; Buenos Aires, Argentina).

126 Plaque assays were performed as previously described (Garcia-Nunez et al., 2010). Briefly, cells 127 were infected with serial dilutions of virus and incubated for $48 \mathrm{~h}$ at $37^{\circ} \mathrm{C}$ with $5 \% \mathrm{CO}_{2}$.

128 Monolayers were fixed with 4\% formaldehyde and stained with crystal violet, and the number 129 and area of individual plaques $(\mathrm{n} \geq 18)$ were determined by using ViralPlaque (Cacciabue, Curra $130 \&$ Gismondi, 2019). Assays were performed in duplicate. 
131 Virus titers were determined in BHK-21 cells as described in (Gismondi et al., 2014) .

132

1332.3 Prediction of RNAi target sequences

134 Target sequences within FMDV 3Dpol coding region (nucleotides 6681-8093 of FMDV A01L)

135 were predicted with RNAxs software (Tafer et al., 2008). This program is freely available at

136 http://rna.tbi.univie.ac.at/cgi-bin/RNAxs/RNAxs.cg. It relies on several siRNA design criteria,

137 namely siRNA strand selection (using both sequence and energy-based rules), self-folding (the

138 minimal free folding-energy of the guide strand), free-end (number of paired nucleotides among

139 the first four at the 5' end and the 3'end of the guide strand) and most importantly accessibility

140 (probability that a region of predefined length is free of base pairing in thermodynamic

141 equilibrium). The program (accessed by October 2014) was run with default values for siRNA.

142 The output file of RNAxs includes a number of putative target sequences of $19 \mathrm{nt}$. To adapt the

143 prediction software to $21 \mathrm{nt}$ target sequences, 2 additional nucleotides were incorporated to each

144 designed small RNA (see Results). Lastly, small RNAs targeting 3Dpol sequence 1162-1182

145 were also used since this region showed high flexibility in a local SHAPE-directed RNA

146 structure obtained at our laboratory (Supplementary Figure 1 and unpublished results).

147 Moreover, it was previously demonstrated that this stretch of FMDV O/CHA/99 can be

148 successfully targeted by RNAi (Gu et al., 2014). In addition, a plasmid encoding a control

149 sequence not expected to target any mammalian mRNA, EGFP or FMDV was used as a negative 150 control.

151

152 2.4 Plasmids

Peer] reviewing PDF | (2020:08:51784:2:0:NEW 5 Mar 2021) 
153

154 155

2.4.1 shRNA encoding plasmids

RNA polymerase III U6 promoter including a BbsI site at the 3' end was amplified by PCR and cloned within plasmid TOPO pCR2.1 (Invitrogen, Carlsbad, USA) to create plasmid pTOPO-U6.

Complementary single-stranded DNA oligonucleotides encoding shRNA against predicted target sequences (Table 1; Supplementary Table 1) were synthesized (Macrogen, Seoul, Korea), annealed and cloned into the BbsI restriction site in pTOPO-U6. The resulting plasmids were named pshRNA ${ }_{\mathrm{FMDV}}$, where FMDV is the target sequence ID.

\subsubsection{Pre-amiRNA encoding plasmids}

Individual pre-amiRNA coding sequences were cloned into into pcDNA ${ }^{\circledR} 6.2-\mathrm{GW} / \mathrm{miR}$ vector (BLOCK-iT Pol II miR RNAi expression vector kit, Invitrogen, Carlsbad, USA) as described in (Gismondi et al., 2014) . The resulting plasmids were named pamiRNA ${ }_{F M D V}$, where FMDV is the target sequence ID.

Dual amiRNA $\mathrm{FMDV}$-expressing plasmids were assembled from individual pamiRNA $\mathrm{FMDV}_{\mathrm{F}}$ according to the manufacturer's instructions (BLOCK-iT Pol II miR RNAi expression vector kit, Invitrogen, Carlsbad, USA) Briefly, donor pamiRNA ${ }_{F M D V}$ DNA was digested with BamHI and

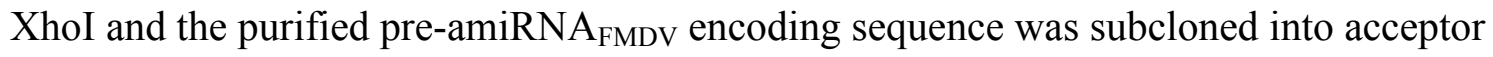

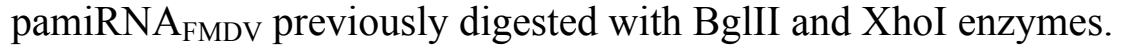

\subsubsection{Enhanced green fluorescent protein (EGFP) reporter plasmids}

A reporter plasmid encoding the EGFP gene fused to the complete FMDV 3Dpol-coding sequence (pEGFP.3D) was constructed. To this end, the EGFP coding sequence was amplified 
174 by PCR using oligonucleotides EGFPfor and EGFPrev (Supplementary Table 1) and cloned

175 between KpnI and EcoRI restriction sites in pcDNA3 vector (Invitrogen, Carlsbad, USA). In

176 addition, the FMDV A01L 3Dpol-coding sequence was amplified by PCR and cloned

177 immediately downstream of the EGFP termination codon.

178 All recombinant vectors were confirmed by automated sequencing.

Pre-amiRNA $A_{F M D V}$ and shRNA ${ }_{F M D V}$-encoding plasmids were transfected into $95 \%$ confluent

BHK-21 cells seeded in 48-well culture plates using PolyAr reagent (School of Biochemistry and Pharmacy, University of Buenos Aires), according to manufacturer's instructions. Briefly, cells were washed with serum-free DMEM, and incubated with $125 \mu 1$ of transfection mixture containing 900 ng DNA, $1 \mu$ l PolyAr and DMEM for $4 \mathrm{~h}$ at $37^{\circ} \mathrm{C}$ and $5 \% \mathrm{CO}_{2}$. Transfected cells were washed three times with sterile $1 \mathrm{X}$ PBS and incubated for additional time in DMEM $10 \% \mathrm{FBS}$ at $37^{\circ} \mathrm{C}$ and $5 \% \mathrm{CO}_{2}$.

When indicated, $1.3 \times 10^{6} \mathrm{BHK}-21$ cells were electroporated with $6 \mu \mathrm{g}$ DNA using GenePulser XCell (BioRad, Hercules, CA, USA). Electroporation conditions included 1 pulse at $280 \mathrm{~V}$ for $25 \mathrm{~ms}$ in 4mm-cuvettes. Electroporated cells were seeded in 48-well culture plates and incubated in DMEM $10 \% \mathrm{FBS}$ at $37^{\circ} \mathrm{C}$ and $5 \% \mathrm{CO}_{2}$ for $18 \mathrm{~h}$ prior to $\mathrm{FMDV}$ infection. 


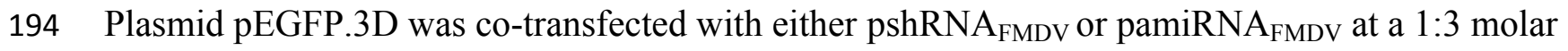

195 ratio using PolyAr as described above. At $24 \mathrm{~h}$ postransfection, EGFP expression was evaluated

196 by fluorescence microscopy in a Zeiss microscope. In addition, transfected cells were washed 2

197 times with 1X PBS, trypsinized and fixed in 1\% formaldehyde for 20 min on ice. After 2 washes

198 in $1 \mathrm{X}$ PBS 2mM EDTA, EGFP expression was quantified by flow cytometry. A BD

199 FACSCalibur and FlowJo ${ }^{\text {TM }}$ Software (Becton, Dickinson and Company; 2019) were used to

200 measure frequency and median fluorescence intensity of EGFP+ cells. Of the 10,000 events

201 evaluated per sample, only those with the forward scatter and side scatter properties of viable

202 cells were used in measurements of EGFP fluorescence intensity.

203

2042.7 Establishment of transgenic cell lines

205 At 24 h post-transfection with pamiRNAFMDV, cells were subcultured in DMEM medium

206 containing 10\% FBS and $7 \mu \mathrm{g} / \mathrm{ml}$ blasticidin S (Invitrogen, Carlsbad, USA). Transgenic

207 polyclonal cell lines were established by consecutive passages in DMEM 10\% FBS and $7 \mu \mathrm{g} / \mathrm{ml}$

208 blasticidin S.

209 Polyclonal cell lines were further cloned by limiting dilution as described in (Gismondi et al.,

210 2014). Transgenesis was confirmed by PCR using oligonucleotides miRNAseq for and

211 miRNAseq rev (Supplementary Table 1).

212

2132.8 Antiviral activity of small RNAs against FMDV 
214 Electroporated cells were infected for $60 \mathrm{~min}$ at a multiplicity of infection (moi) of 3-5. Cells

215 were washed with 1X PBS pH 5.0 on ice to inactivate unabsorbed virus. After restitution of

216 physiological $\mathrm{pH}$, cells were incubated in DMEM 2\% FBS and $25 \mathrm{mM}$ HEPES pH 7.5 at $37^{\circ} \mathrm{C}$

217 in a 5\% $\mathrm{CO}_{2}$ atmosphere. At indicated times post-infection, cells were lysed by three consecutive

218 freeze-thaw cycles and the amount of viral particles was measured by the $\mathrm{TCID}_{50}$ method as

219 mentioned above. Percentage of inhibition was calculated from the following equation: $\mathrm{P}=((\mathrm{A}-$

220 B)*100)/A, where A was viral titer of cell lines expressing miRneg infected with FMDV

$221 \mathrm{~A} / \mathrm{Arg} / 01$, and $\mathrm{B}$ was viral titer of cell lines expressing amiRNA ${ }_{\mathrm{FMDV}}$ infected with FMDV

$222 \mathrm{~A} / \mathrm{Arg} / 01$. Experiments were performed in duplicate.

223 Stable cell lines expressing amiRNA ${ }_{F M D V}$ were infected at an moi of 0.001 and incubated in

224 DMEM 2\% FBS and $25 \mathrm{mM}$ HEPES pH 7.5 at $37^{\circ} \mathrm{C}$ and $5 \% \mathrm{CO}_{2}$. At different times post-

225 infection, the virus present in supernatants was titrated by the $\mathrm{TCID}_{50}$ method.

227 2.9 Statistical analysis

228 Student's $t$ test was used to compare mean viral titers in different samples and mean plaque areas.

229 Differences in median fluorescence intensity in cells transfected with different plasmids were

230 compared using Kruskal-Wallis test. A p value $<0.05$ was considered as statistically significant.

3. Results 
234 Target sequences were predicted within FMDV A01L 3Dpol-coding sequence with RNAxs

235 software (Tafer et al., 2008), which applies several prediction parameters including RNA

236 accessibility. A total number of 178 target sequences were predicted along 3D (Figure 1, blue

237 areas; Supplementary Table 2), with top 50 predicted targets concentrated in discrete regions

238 (Figure 1, red areas).

239 Next, an additional screening of the target sequences ranking in the top positions of the RNAxs

240 output was performed to take into account the prediction rules proposed by Low et al. (Low et

241 al., 2012). Firstly, $19 \mathrm{nt}$ target sequences were extended to $21 \mathrm{nt}$ in order to adapt RNAxs

242 prediction to amiRNA-compatible length. Then, the resulting target sequences were analyzed in

243 terms of the accessibility window proposed by Low et al., i.e., sequences showing a less

244 structured stretch downstream of the $21 \mathrm{nt}$ target region were favored. Lastly, the total free

245 energy change ( $\Delta$ Gtotal) of target:siRNA duplex formation was calculated with OligoWalk

246 program (Reuter \& Mathews, 2010) (Table 1). After applying these rules, sequences 444-464,

247 290-310 and 1055-1075 were selected (termed 444; 290 and 1055; Figure 1, Table 1 and

248 Supplementary Figure 1). The selected target sequences ranked in the first positions of the

249 RNAxs output. Indeed, in some cases the $21 \mathrm{nt}$ target sequences included more than one $19 \mathrm{nt}$

250 predicted target sequence (Table 1). Of note, sequences 290 and 444 displayed a $\Delta$ Gtotal value

251 higher than the one recommended by Low et al. (-25 Kcal/mol). Regarding the accessibility

252 window, no target sequence showed the expected accessible 13 nt window starting at position 14

253 (Supplementary Figure 1a). However, all sequences were located in the region of high linear

254 correlation between RNA structure and shRNA efficacy described by these authors

255 (Supplementary Figure 1B). 
256 Finally, conservation of the selected target sequences among prototypic strains of the South

257 American serotype pool was evaluated (Supplementary Figure 1C). Sequences 290, 444 and

2581055 showed some polymorphisms between serotypes, which were distributed mainly outside

259 the seed region.

260

2613.2 Silencing efficacy of shRNAs and amiRNAs targeting selected FMDV sequences

262 A reporter assay was conducted to test the silencing efficacy of different RNAi mediators

263 targeting the selected sequences. To this end, a plasmid encoding EGFP fused to the 3Dpol-

264 coding sequence of FMDV A/Arg/01 was co-transfected with plasmids encoding either shRNAs

265 or amiRNAs targeting each individual FMDV sequence. Short hairpin RNAs targeting sequences

266290,444 and 1055 induced a significant reduction of EGFP expression in co-transfected cells, as

267 determined by fluorescence microscopy and flow cytometry (Figure 2A and Supplementary

268 Figure 2A). In turn, amiRNAs targeting single FMDV sequences also exhibited significant

269 silencing activity (Figure 2B and Supplementary Figure 2B).

270 To evaluate the impact of dual amiRNA ${ }_{F M D V}$ expression on silencing activity in reporter assays,

271 pre-amiRNA 290 , pre-amiRNA 444 or pre-amiRNA 1162 were subcloned in pamiRNA 1055 to obtain

272 bicistronic expression constructs and the resulting plasmids were co-transfected with pEGFP.3D

273 in BHK-21 cells. As shown in Figure 2C, dual expression of amiRNAs did not enhance silencing 274 of the reporter mRNA.

2763.3 Antiviral activity of shRNA ${ }_{\mathrm{FMDV}}$ and amiRNA $\mathrm{FMDV}$ 
277 Next, we evaluated the antiviral activity of shRNAs and amiRNAs against FMDV in cultured

278 cells. To this end, BHK-21 cells were electroporated with the small RNA-expressing plasmids,

279 and after $16 \mathrm{~h}$ the cells were infected with FMDV at high multiplicity of infection. Viral titers

280 were determined at different times post infection to assess the effect of small RNAs on viral

281 growth. As listed in Table 2, inhibition of viral growth was evidenced at 5 hours post-infection

282 (hpi) in cells expressing small RNAs targeting regions 290, 444 and 1055. Interestingly,

283 shRNA $_{290}$ induced a 2-fold reduction of FMDV replication, whereas shRNA 444 and shRNA 1055

284 induced a more pronounced decrease in viral titers at this time point. Conversely, there was a

285 higher inhibition of viral growth in cells expressing amiRNA 290 ( $>10$-fold) than in cells

286 electroporated with pamiRNA 444 and pamiRNA 1055 . Again, dual expression of amiRNAs

287 targeting different FMDV sequences did not improve the silencing activity of each individual

288 amiRNA (Table 2). Of note, the silencing efficacy of small RNAs determined previously in our

289 reporter assays was confirmed in the context of viral replication except for shRNA ${ }_{1162}$, which did 290 not affect FMDV replication significantly.

3.4 Stable expression of amiRNAs against FMDV

293

294

295

296

297

Electroporation of DNA is a well-established method that yields a high frequency of

transformants (Potter, 2003); however, transfection efficiency is not 100\%. We hypothesized that electroporation of plasmids expressing small RNAs could lead to a population of non-transfected cells that, when infected, would contribute to FMDV replication. To circumvent this possibility, stably-transformed cell lines expressing amiRNA 290 and amiRNA 1055 were established and 
298 characterized in terms of antiviral efficacy. In this case, only amiRNAs were evaluated since

299 they exhibit less toxicity than shRNAs (Boudreau, Monteys \& Davidson, 2008).

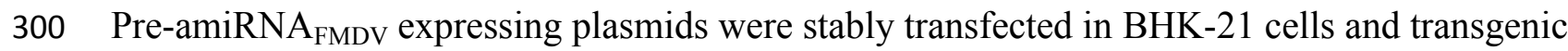

301 polyclonal cell lines were selected by blasticidin resistance. Mature amiRNA expression was

302 confirmed by RT-stem loop qPCR (Supplementary Figure 4A). After several passages, cells

303 were infected with FMDV A/Arg/01 and cultures were monitored to evaluate the development of

304 cytopathic effect. At 48 hpi, control cell monolayers (non-transfected BHK-21 cells and cells

305 stably transfected with a non-specific amiRNA) were completely detached due to FMDV

306 infection. In contrast, infection of cell lines stably expressing amiRNA 290 and amiRNA 1055

307 caused partial cytopathic effect, with a significant proportion of cells still adherent to the culture

308 surface (Figure 3A). This effect was also evidenced in lysis plaques assays, where FMDV

309 infection rendered less plaques of smaller dimensions in cell lines expressing amiRNA 290 and

310 amiRNA $_{1055}$ than in control cells (Figure 3B). However, viral titers determined at different times

311 post-infection in supernatants of amiRNA 290 and amiRNA 1055 polyclonal cells did not differ

312 significantly from control cells except for amiRNA 290 cells at 18 hpi (Figure 3C). Together, these

313 results suggest a transient amiRNA-mediated constraint on FMDV replication in transgenic cell

314 lines.

315 Next, polyclonal cell lines expressing amiRNA 290 and amiRNA 1055 were cloned by limiting

316 dilution to consider the potential variability in transgene expression associated with multiple

317 transgene insertion events during the establishment and selection of stable transformants. The

318 resulting cell lines were infected with FMDV A/Arg/01 and their ability to control viral growth

319 was assessed by plaque assay (data not shown). Cloned cell lines showing less or smaller plaques

320 than control cells (BHK-21 and miRneg-expressing cells) were selected for further analysis. 
321 Selected cell lines ( $\mathrm{n}=4$ for $\operatorname{amiRNA}_{290}$ and $\mathrm{n}=3$ for amiRNA $\left.{ }_{1055}\right)$ were infected with FMDV

$322 \mathrm{~A} / \mathrm{Arg} / 01$ and viral growth was determined by viral titration in supernatants at different times

323 post-infection. As shown in Figure 4, viral growth was delayed in the majority of the cloned cell

324 lines, supporting the potential use of amiRNAs as antivirals. However, at 48 hpi, the viral titers

325 in supernatants of infected transgenic cells did not differ from control cells. Moreover, direct

326 sequencing of a 250-300 nt region encompassing the target site did not show any mutation that

327 could account for virus escape (Supplementary Figure 5).

\section{Discussion}

To develop an antiviral strategy against FMDV based on RNAi, the 3Dpol-coding sequence of

FMDV A/Arg/01 was used to predict target sites. This region of the viral genome is highly conserved within FMDV serotypes. Moreover, the essential role played by the protein product (3Dpol) in the viral replication cycle makes it an attractive target for RNA interference. As shown in the reporter assays, the predicted targets were silenced efficiently independently of the RNAi mediator used, supporting the feasibility of the algorithm applied for target selection. Our results are in accordance with previous data indicating that the $3 \mathrm{D}$ region of the FMDV genome is a suitable target for RNAi (Pengyan et al., 2008; Du et al., 2011; Gu et al., 2014; Basagoudanavar et al., 2019) and they represent a step forward in the identification of new target sequences within FMDV, specifically in serotype A, that had been less explored in previous works.

341 It is well known that target accessibility may limit RNAi efficacy (Westerhout, 2005; Tafer et al., 342 2008; Low et al., 2012; Gismondi et al., 2014). In a paper on HIV inhibition by RNAi, Low et al. 
343 (2012) analyzed the impact of target RNA structure and target:siRNA duplex thermodynamics

344 on shRNA efficacy. They found a maximal negative correlation for a 13-nt window that starts at

345 position 14 of the target RNA and extends over 7 nucleotides downstream of the "seed region"

346 binding site. In the context of RNAi, a negative correlation implies that maximal shRNA

347 inhibition is achieved for less structured target RNAs. Moreover, $\Delta$ Gtotal of target:siRNA duplex

348 formation strongly correlated with shRNA inhibition. Thus, in this work RNA accessibility was

349 used as a central criterion for target selection.

350 The available programs for prediction of RNAi targets can be classified as first- and second-

351 generation algorithms. The latter are based not only on a number of selection criteria but also

352 they were optimized to include results from real RNAi experiments to validate their predictions

353 (Griger \& Tisminetzky, 1984; Devaney et al., 1988; Filhol et al., 2012). In the present work we

354 used a first-generation RNAi target selection program but we also included the experimental data

355 derived from the work by Low et al (2012) to predict RNAi targets. Interestingly, none of the

356 selected regions had been targeted previously in other similar studies with other FMDV

357 serotypes (Chen et al., 2004; Kahana et al., 2004; Mohapatra et al., 2005; Du et al., 2011).

358 Furthermore, our analysis with RNAxs did not retrieve the targets selected by other authors

359 within the 15 best ranking sequences or it did even not predict them at all. This may indicate

360 that, despite sequence conservation between FMDV serotypes in the 3D region, accessibility of

361 specific target sequences may not be equivalent between serotypes due to structural constraints

362 in the context of the whole genome, as we previously reported.

363 Indeed, conservation of the target sequence among different viral strains should be considered

364 during the development of RNAi-based antiviral tools (Mcintyre et al., 2009). In this work,

365 selected sequences 290, 444 and 1055 showed partial conservation among South American 
366 serotypes; however, the sequences used for small RNA design were the most frequent at

367 polymorphic sites (Supplementary Figure 1C). Interestingly, other stretches within 3D showing

$368100 \%$ conservation were not predicted in the top-ranking target sites by RNAxs software,

369 suggesting these sequences may not be accessible. Initial reports on determinants of

370 miRNA:mRNA recognition highlighted the existence of two categories of target sites. 5,

371 dominant sites, which are strongly complementary to the miRNA 5' end, need little or no pairing

372 of the miRNA 3' end to achieve silencing. On the other hand, miRNAs with partial

373 complementarity at the 5' end require strong 3' compensatory pairing for function (Brennecke et

374 al., 2005; Bartel, 2009). Thus, the existence of polymorphisms in the target sequence should not

375 preclude miRNA efficacy against different FMDV serotypes. Moreover, potentially reduced

376 RNAi due to weak amiRNA:target RNA pairing at polymorphic sites could be circumvented by

377 co-expression of multiple amiRNAs targeting different target sequences of the viral genome.

378 Interestingly, the shRNA directed against sequence 1162 was effective in the reporter assay and

379 the amiRNA trended to perform in a similar way, although with no statistical significance.

380 Contrarily, shRNA 1162 did not affect FMDV replication significantly, whereas amiRNA 1162

381 showed moderate antiviral activity. This target region was selected as a putative positive control,

382 since the same sequence of FMDV serotype O/CHA/99 (Supplementary Figure 3) had been

383 efficiently silenced by an shRNA in a previous paper (Gu et al., 2014). Noteworthy, as

384 mentioned before, this region was not predicted as a suitable RNAi target by RNAxs software. It

385 may be argued that shRNA 1162 did not work due to structural constraints of the target region in

386 the context of the whole viral genome, which were not present in the reporter mRNA. However,

387 the results obtained with amiRNA 1162 indicate that target site accessibility should not be limiting

388 shRNA $_{1162}$ efficacy. Alternatively, it is possible that this target region is not completely 
389 accessible to the mature small RNAs, leaving only some nucleotides available for small

390 RNA:target recognition. In this case, pairing of the seed region of amiR $\mathrm{R}_{1162}$ would be sufficient

391 for activation of the RNAi machinery, whereas incomplete pairing between shRNA 1162 and its

392 target RNA would not lead to RNA silencing. In addition, since it has been shown that siRNA

393 derived from shRNA precursors are more heterogeneous in cleavage sites and length than small

394 RNAs originating from miRNA precursors (Maczuga et al., 2013), differences in shRNA and

395 pre-amiRNA structures and processing that may lead to slightly different mature small RNAs

396 with singular silencing efficacy cannot be ruled out.

397 Short hairpin RNAs are highly transcribed from RNA polymerase III promoters such as the U6

398 promoter used in this study. In addition, it has been demonstrated that shRNAs are more potent

399 than amiRNAs (Boudreau, Monteys \& Davidson, 2008) when they are expressed from the same

400 promoter. However, shRNAs exhibit higher cellular toxicity than amiRNAs since the high levels

401 of shRNA precursors produced in a cell may saturate the endogenous miRNA processing

402 machinery (Boudreau, Martins \& Davidson, 2009). In our reporter assays, shRNAs and

403 amiRNAs were transcribed from different promoters (U6 and CMV, for RNA polymerases III

404 and II, respectively). and we did not detect any difference in cell viability between cells

405 transfected with pshRNA ${ }_{F M D V}$ and cells transfected with pamiRNA ${ }_{F M D V}$ as determined by flow

406 cytometry.

407 Our results show that amiRNAs induce a significant inhibition of viral replication in cultured

408 cells; however, silencing of FMDV RNA is not complete leading to sustained viral replication.

409 Notably, amiRNAs were poorly expressed in the majority of cloned cell lines as compared to

410 polyclonal cells (Supplementary Figure 4B). Thus, the lack of sustained viral inhibition may be

411 associated with insufficient amiRNA expression. However, a $\sim 24 \mathrm{~h}$ delay in viral replication has

Peer] reviewing PDF | (2020:08:51784:2:0:NEW 5 Mar 2021) 
412 been also reported by de los Santos et al (de los Santos et al., 2005), Chen et al (Chen et al.,

413 2004) and Lv et al (Lv et al., 2009) using shRNAs or siRNAs. Conversely, Kahana et al (Kahana

414 et al., 2004) showed a sustained inhibition of FMDV infection in BHK-21 cells transfected with

415 siRNAs against FMDV; however, the authors only analyzed viral replication for $24 \mathrm{~h}$. In turn, by

416 using an siRNA transfected in BHK-21 cells, Mohapatra et al (Mohapatra et al., 2005) reported a

417 reduction of viral titer of $>99 \%$ at $24 \mathrm{hpi}$ and $>87 \%$ at $48 \mathrm{hpi}$. The transitory character of viral

418 inhibition may be explained by several factors. First of all, it should be kept in mind that in the

419 infected cell the FMDV RNA is associated with multiple cellular and viral proteins during the

420 processes of genome translation and replication. Thus, target sequences should not be expected

421 to be continuously accessible for the silencing complexes during the whole FMDV replication

422 cycle. In addition, it is well known that FMDV proteases induce a rapid shut-off of cellular

423 transcription and cap-dependent translation (Kim et al., 2008; Qureshi, Thakur \& Kumar, 2013).

424 Given that amiRNAs are expressed from the strong CMV promoter, it is expected that pre-

425 amiRNA transcription is also inhibited in infected cells in the context of viral replication. Thus,

426 the incomplete inhibition of FMDV replication by amiRNAs may be explained by a FMDV-

427 mediated reduction in transgene expression.

428 Other authors have demonstrated that simultaneous expression of multiple small RNAs directed 429 against different regions of the same target RNA can increase silencing efficacy (Kahana et al., 430 2004; Brake et al., 2008; Liu et al., 2008; Kim et al., 2010; Saha et al., 2016). This approach

431 becomes particularly relevant when the target RNA is a viral genome, since multiple small

432 RNAs acting on the same genome reduce the chance of emergence of escape mutants thus

433 increasing safety. However, in this work dual amiRNA expression did not enhance silencing of

434 the reporter mRNA or the complete FMDV genome as compared to individual amiRNA 
435 expression. Similar results have been reported by other authors using amiRNAs against FMDV

436 (Basagoudanavar et al., 2019). Noteworthy, amiRNA expression did not differ significantly in

437 cells stably transfected with monocistronic or bicistronic vectors (Supplementary Figure 4A).

438 Further research is needed to determine whether it is due to structural constraints of the target

439 RNA or to the intrinsic regulation of the RNAi machinery.

\section{Conclusions}

442

443

444

445

446

447

448

449

450

451

452

453

In light of the results obtained, we conclude that the selection algorithm proved to be highly accurate in the identification of target sequences. FMDV replication may be transiently controlled by amiRNAs targeting 3D. Ongoing work in our laboratory is aimed at finding new targets in other genomic regions of FMDV to develop additional amiRNAs that could potentiate the antiviral effects observed in the present work. Our results highlight the potential application of amiRNAs as antiviral tools which may complement vaccination, specifically to prevent the spread of the disease during outbreaks in endemic or non-endemic countries.

\section{Acknowledgements}

A.C. is a doctoral fellow and M.C of the National Research Council (CONICET) at the University of Luján, Argentina. M.C. is a postdoctoral fellow of CONICET. S.A., O.T. and M.I.G. are members of CONICET Research Career Program. 
454 We thank María José Mónaco and Osvaldo Zábal for technical assistance and Juan Manuel

455 Schammas and Alfredo Perea for their support during our work in the BSL4-OIE facilities at the 456 Institute of Virology-INTA.

457

458

\section{References}

460

461

462

463

464

465

466

467

468

469

470

471

472

473

474

475

476

Andino R, Domingo E. 2015. Viral quasispecies. Virology 479-480:46-51. DOI: 10.1016/j.virol.2015.03.022.

Bartel DP. 2009. MicroRNAs: Target Recognition and Regulatory Functions. Cell 136:215-233. DOI: 10.1016/j.cell.2009.01.002.

Basagoudanavar SH, Ranjitha HB, Hosamani M, Kolangath SM, Selvan RPT, Sreenivasa BP, Saravanan P, Sanyal A, Venkataramanan R. 2019. Efficient inhibition of foot-and-mouth disease virus replication in vitro by artificial microRNA targeting 3D polymerase. Acta Virol 63:475-479. DOI: 10.4149/av_2019_407.

Boudreau RL, Martins I, Davidson BL. 2009. Artificial microRNAs as siRNA shuttles: improved safety as compared to shRNAs in vitro and in vivo. Mol Ther 17:169-75. DOI: 10.1038/mt.2008.231

Boudreau RL, Monteys AM, Davidson BL. 2008. Minimizing variables among hairpin-based RNAi vectors reveals the potency of shRNAs. $R N A$ 14:1834-44. DOI: 10.1261/rna.1062908.

Brake O ter, Hooft K ’t, Liu YP, Centlivre M, Jasmijn von Eije K, Berkhout B. 2008. Lentiviral Vector Design for Multiple shRNA Expression and Durable HIV-1 Inhibition. Molecular Therapy 16:557-564. DOI: 10.1038/sj.mt.6300382. 
477 Brennecke J, Stark A, Russell RB, Cohen SM. 2005. Principles of MicroRNA-Target

$478 \quad$ Recognition. PLoS Biology 3:e85. DOI: 10.1371/journal.pbio.0030085.

479 Cacciabue M, Curra A, Gismondi MI. 2019. ViralPlaque: a Fiji macro for automated assessment 480 of viral plaque statistics. PeerJ 7:e7729. DOI: 10.7717/peerj.7729.

481 Carthew RW, Sontheimer EJ. 2009. Origins and Mechanisms of miRNAs and siRNAs. Cell 482 136:642-55. DOI: 10.1016/j.cell.2009.01.035.

483

484

485

486

487

488

489

490

491

492

493

494

495

496

497

498

Castanotto D, Rossi JJ. 2009. The promises and pitfalls of RNA-interference-based therapeutics. Nature 457:426-33. DOI: 10.1038/nature07758.

Chen C. 2005. Real-time quantification of microRNAs by stem-loop RT-PCR. Nucleic Acids Research 33:e179-e179. DOI: 10.1093/nar/gni178.

Chen W, Yan W, Du Q, Fei L, Liu M, Ni Z, Sheng Z, Zheng Z. 2004. RNA interference targeting VP1 inhibits foot-and-mouth disease virus replication in BHK-21 cells and suckling mice. J Virol 78:6900-7. DOI: 10.1128/JVI.78.13.6900-6907.2004.

Deng S, Li G, Yu K, Tian X, Wang F, Li W, Jiang W, Ji P, Han H, Fu J, Zhang X, Zhang J, Liu Y, Lian Z, Liu G. 2017. RNAi combining Sleeping Beauty transposon system inhibits ex vivo expression of foot-and-mouth disease virus VP1 in transgenic sheep cells. Sci Rep 7:10065. DOI: 10.1038/s41598-017-09302-1.

Devaney MA, Vakharia VN, Lloyd RE, Ehrenfeld E, Grubman MJ. 1988. Leader protein of footand-mouth disease virus is required for cleavage of the p220 component of the capbinding protein complex. J Virol 62:4407-9.

Dicara D, Burman A, Clark S, Berryman S, Howard MJ, Hart IR, Marshall JF, Jackson T. 2008. Foot-and-mouth disease virus forms a highly stable, EDTA-resistant complex with its 
499

500

501

502

503

504

505

506

507

508

509

510

511

512

513

514

515

516

517

518

519

520

521

principal receptor, integrin alphavbeta6: implications for infectiousness. J Virol 82:153746. DOI: 10.1128/JVI.01480-07.

Domingo E, Escarmís C, Baranowski E, Ruiz-Jarabo CM, Carrillo E, Núñez JI, Sobrino F. 2003. Evolution of foot-and-mouth disease virus. Virus Research 91:47-63. DOI: 10.1016/s0168-1702(02)00259-9.

Domingo E, Sheldon J, Perales C. 2012. Viral Quasispecies Evolution. Microbiology and Molecular Biology Reviews 76:159-216. DOI: 10.1128/MMBR.05023-11.

Du J, Gao S, Luo J, Zhang G, Cong G, Shao J, Lin T, Cai X, Chang H. 2011. Effective inhibition of foot-and-mouth disease virus (FMDV) replication in vitro by vector-delivered microRNAs targeting the 3D gene. Virol J 8:292. DOI: 10.1186/1743-422X-8-292.

Filhol O, Ciais D, Lajaunie C, Charbonnier P, Foveau N, Vert JP, Vandenbrouck Y. 2012. DSIR: assessing the design of highly potent siRNA by testing a set of cancer-relevant target genes. PLoS One 7:e48057. DOI: 10.1371/journal.pone.0048057.

Fischer SEJ. 2015. RNA Interference and MicroRNA-Mediated Silencing. Curr Protoc Mol Biol 112:26 1 1-26 1 5. DOI: 10.1002/0471142727.mb2601s112.

Garcia-Nunez S, Konig G, Berinstein A, Carrillo E. 2010. Differences in the virulence of two strains of Foot-and-Mouth Disease Virus Serotype A with the same spatiotemporal distribution. Virus Res 147:149-52. DOI: 10.1016/j.virusres.2009.10.013.

Gismondi MI, Ortiz XP, Curra AP, Asurmendi S, Taboga O. 2014. Artificial microRNAs as antiviral strategy to FMDV: structural implications of target selection. J Virol Methods 199:1-10. DOI: 10.1016/j.jviromet.2013.12.016.

Golde W, Pacheco J, Duque H, Doel T, Penfold B, Ferman G, Gregg D, Rodriguez L. 2005. Vaccination against foot-and-mouth disease virus confers complete clinical protection in 
522

523

524

525

526

527

528

529

530

531

532

533

534

535

536

537

538

539

540

541

542

543

544

7 days and partial protection in 4 days: Use in emergency outbreak response. Vaccine 23:5775-5782. DOI: 10.1016/j.vaccine.2005.07.043.

Griger PR, Tisminetzky SG. 1984. Histone H3 modification in BHK cells infected with foot-andmouth disease virus. Virology 136:10-19. DOI: 10.1016/0042-6822(84)90243-5.

Grubman MJ, Baxt B. 2004. Foot-and-mouth disease. Clin Microbiol Rev 17:465-93. DOI: 10.1128/cmr.17.2.465-493.2004.

Gu YX, Gao ZL, Zhou JH, Zhang J, Liu YS. 2014. Establishment and evaluation of stable cell lines inhibiting foot-and-mouth disease virus by RNA interference. Biomed Res Int 2014:109428. DOI: 10.1155/2014/109428.

Hu S, Qiao J, Fu Q, Chen C, Ni W, Wujiafu S, Ma S, Zhang H, Sheng J, Wang P, Wang D, Huang J, Cao L, Ouyang H. 2015. Transgenic shRNA pigs reduce susceptibility to foot and mouth disease virus infection. Elife 4:e06951. DOI: 10.7554/eLife.06951.

Jiao Y, Gong X, Du J, Liu M, Guo X, Chen L, Miao W, Jin T, Chang H, Zeng Y, Zheng Z. 2013. Transgenically mediated shRNAs targeting conserved regions of foot-and-mouth disease virus provide heritable resistance in porcine cell lines and suckling mice. Vet Res 44:47. DOI: $10.1186 / 1297-9716-44-47$.

Kahana R, Kuznetzova L, Rogel A, Shemesh M, Hai D, Yadin H, Stram Y. 2004. Inhibition of foot-and-mouth disease virus replication by small interfering RNA. J Gen Virol 85:32133217. DOI: 10.1099/vir.0.80133-0.

Kim SM, Lee KN, Lee SJ, Ko YJ, Lee HS, Kweon CH, Kim HS, Park JH. 2010. Multiple shRNAs driven by U6 and CMV promoter enhances efficiency of antiviral effects against foot-and-mouth disease virus. Antiviral Res 87:307-17. DOI: 10.1016/j.antiviral.2010.06.004. 
545 Kim SM, Lee KN, Park JY, Ko YJ, Joo YS, Kim HS, Park JH. 2008. Therapeutic application of 546 RNA interference against foot-and-mouth disease virus in vitro and in vivo. Antiviral Res 80:178-84. DOI: 10.1016/j.antiviral.2008.06.001.

548 Lawrence P, Rai D, Conderino JS, Uddowla S, Rieder E. 2016. Role of Jumonji C-domain 549 containing protein 6 (JMJD6) in infectivity of foot-and-mouth disease virus. Virology 492:38-52. DOI: 10.1016/j.virol.2016.02.005.

551 Liu YP, Haasnoot J, ter Brake O, Berkhout B, Konstantinova P. 2008. Inhibition of HIV-1 by

552

553

554

555

556

557 558

559

560

561

562

563

564

565

566 multiple siRNAs expressed from a single microRNA polycistron. Nucleic Acids Res 36:2811-24. DOI: 10.1093/nar/gkn109.

Low JT, Knoepfel SA, Watts JM, ter Brake O, Berkhout B, Weeks KM. 2012. SHAPE-directed discovery of potent shRNA inhibitors of HIV-1. Mol Ther 20:820-8. DOI: 10.1038/mt.2011.299.

Lv K, Guo Y, Zhang Y, Wang K, Li K, Zhu Y, Sun S. 2009. Transient inhibition of foot-andmouth disease virus replication by siRNAs silencing VP1 protein coding region. Res Vet Sci 86:443-52. DOI: 10.1016/j.rvsc.2008.10.011.

Maczuga P, Lubelski J, van Logtenstein R, Borel F, Blits B, Fakkert E, Costessi A, Butler D, van Deventer S, Petry H, Koornneef A, Konstantinova P. 2013. Embedding siRNA sequences targeting apolipoprotein B100 in shRNA and miRNA scaffolds results in differential processing and in vivo efficacy. Mol Ther 21:217-27. DOI: 10.1038/mt.2012.160.

Mcintyre GJ, Groneman JL, Yu Y-H, Jaramillo A, Shen S, Applegate TL. 2009. 96 shRNAs designed for maximal coverage of HIV-1 variants. Retrovirology 6:55. DOI: 10.1186/1742-4690-6-55. 
567 Mohapatra JK, Sanyal A, Hemadri D, Tosh C, Kumar RM, Bandyopadhyay SK. 2005.

568 Evaluation of in vitro inhibitory potential of small interfering RNAs directed against

569 various regions of foot-and-mouth disease virus genome. Biochem Biophys Res Commun

570 329:1133-8. DOI: 10.1016/j.bbrc.2005.02.080.

571 Pengyan W, Yan R, Zhiru G, Chuangfu C. 2008. Inhibition of foot-and-mouth disease virus

572 replication in vitro and in vivo by small interfering RNA. Virol J 5:86. DOI:

$573 \quad 10.1186 / 1743-422 X-5-86$.

574 Potter H. 2003. Transfection by electroporation. Curr Protoc Mol Biol Chapter 9:Unit 9 3. DOI:

$575 \quad 10.1002 / 0471142727 . m b 0903 s 62$.

576 Qureshi A, Tantray VG, Kirmani AR, Ahangar AG. 2018. A review on current status of antiviral 577 siRNA. Rev Med Virol 28:e1976. DOI: 10.1002/rmv.1976.

578 Qureshi A, Thakur N, Kumar M. 2013. VIRsiRNApred: a web server for predicting inhibition 579 efficacy of siRNAs targeting human viruses. J Transl Med 11:305. DOI: 10.1186/1479$580 \quad 5876-11-305$.

581 Reuter JS, Mathews DH. 2010. RNAstructure: software for RNA secondary structure prediction 582 and analysis. BMC Bioinformatics 11:129. DOI: 10.1186/1471-2105-11-129.

583 Saha A, Bhagyawant SS, Parida M, Dash PK. 2016. Vector-delivered artificial miRNA

584 effectively inhibited replication of Chikungunya virus. Antiviral Res 134:42-49. DOI:

$585 \quad$ 10.1016/j.antiviral.2016.08.019.

586 de los Santos T, Wu Q, de Avila Botton S, Grubman MJ. 2005. Short hairpin RNA targeted to 587 the highly conserved 2B nonstructural protein coding region inhibits replication of 588 multiple serotypes of foot-and-mouth disease virus. Virology 335:222-31. DOI: 10.1016/j.virol.2005.03.001. 
590 Shah PS, Schaffer DV. 2011. Antiviral RNAi: translating science towards therapeutic success. 591 Pharm Res 28:2966-82. DOI: 10.1007/s11095-011-0549-8.

592 Tafer H, Ameres SL, Obernosterer G, Gebeshuber CA, Schroeder R, Martinez J, Hofacker IL. 593 2008. The impact of target site accessibility on the design of effective siRNAs. Nat $594 \quad$ Biotechnol 26:578-83. DOI: 10.1038/nbt1404.

595 Westerhout EM. 2005. HIV-1 can escape from RNA interference by evolving an alternative 596 structure in its RNA genome. Nucleic Acids Research 33:796-804. DOI:

$597 \quad 10.1093 /$ nar/gki220.

598

599

600 


\section{Figure captions}

602

603

604

605

606

607

608

609

610

611

612

613

614

615

616

617

618

619

620

621

Fig. 1 RNAxs results of target prediction within FMDV 3D region. Data are represented as the number of predicted target sequences (coverage) per 3D nucleotide. Total number of predicted sequences (blue areas) and sequences corresponding to the top 50 hits in the RNAxs ranking (red areas) are shown. Target sequences selected for further study are indicated at the top of the corresponding peaks

Fig. 2 Silencing activity of small RNAs directed against FMDV 3D sequences. Co-transfected cells were trypsinized and EGFP expression was analyzed by flow cytometry at 24 hpi as described in Material and Methods. Cells were co-transfected with pEGFP3D and shRNA $A_{F M D V}$

(A), amiRNA $A_{F M D V}(B)$ or dual amiRNA $A_{F M D V}(C)$ expressing plasmids. ${ }^{*} \mathrm{p}<0.05 ; * * \mathrm{p}<0.01$; $* * * \mathrm{p}<0.001$

Fig. 3 Antiviral effect of amiRNAs $s_{\mathrm{FMDV}}$ stably expressed in BHK-21 cells. (A) Cytopathic effect observed in BHK-21 cell lines expressing amiRNA $\mathrm{FMDV}_{\text {and }}$ control group (cell lines expressing miRneg) at $48 \mathrm{~h}$ post-infection. Cellular detachment, rounding, and destruction of the control group were more severe in the control group than in the experimental group (Magnification 100×). (B) Morphology and (C) dimensions of lysis plaques produced by FMDV in amiRNA $_{\mathrm{FMDV}}$-expressing cell lines and control cells. FMDV infection produced less plaques of smaller dimensions in cell lines expressing amiRNA than in control cells. ${ }^{* * *} \mathrm{p}<0.0001$. (D-F) 
622 Viral titers in supernatants of infected cells were determined by the TCID $_{50}$ method at 18 hpi

623 (D), 24 hpi (E) or 48 hpi (F). ${ }^{* *}$ p $<0.01$.

624

625 Fig. 4 Antiviral activity of cloned $\operatorname{amiRNA}_{290}(\mathrm{~A}-\mathrm{C})$ and $\operatorname{amiRNA}_{1055}$ (D-F) cell lines. Cell lines

626 were obtained from polyclonal cell lines by limiting dilution as described in Material and

627 Methods. Cells were infected with FMDV A/Arg/01 at a low moi and viral titers in supernatants

628 at 18 hpi (A, D), 24 hpi (B, E) or 48 hpi (C, F) were determined by end-point dilution. *p<0.05

629 (as compared to miRneg cells, Student's $t$-test).

630

631 Supplementary Fig. 1 (A) Local RNA structure in the proximity of selected target sequences.

632 RNA structure was predicted using RNAstructure software and SHAPE data covering a 518-nt

633 region spanning positions 892-1410 of FMDV 3D. Target sequences are indicated in orange.

634 Colored nucleotides in sequences 1055-1075 and 1162-1182 represent SHAPE reactivity (red:

635 0.7-1.0; orange: 0.3-0.7; black: 0.0-0.3; grey: no reactivity data). Nucleotides are numbered

636 according to their position in 3Dpol-coding sequence. (B) Correlation between length and

637 starting position of the accessibility window and shRNA efficacy for the training dataset

638 described in Low et al. (Mol Therapy 20:820-828. 2012, modified with permission). The white

639 box indicates the optimal accessible window reported by the authors, starting at position 14 and

640 with a length of $13 \mathrm{nt}$. Black boxes represent the target sequences selected in this work (1: 290;

$6412: 444 ; 3: 1055 ; 4: 1162)$. (C) Conservation of selected target sequences among prototypic

642 FMDV strains of endemic pool 7, which includes serotypes A and $\mathrm{O}$ (www.foot-and-mouth.org).

643 GenBamk accession numbers of prototypic strains are AY593768.1, AY593780.1, AY593815.1, 
644 M10975.1. The target sequence derived from FMDV strain A01L used in this study (GenBank

645 accession No. KY404934) is indicated under each logo.

646 Supplementary Fig. 2 Fluorescence microscopy of BHK-21 cells cotransfected with small RNAs

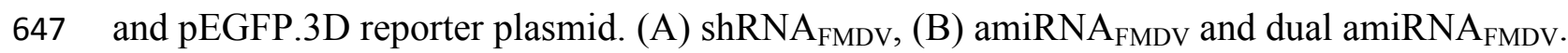

648 Magnification 400X

649

650 Supplementary Fig. 3 Sequence similarity of target sequence 1162 between FMDV A/Arg/01

651 and FMDV O/CHA/99 (GenBank Accession Numbers KY404934 and AF506822, respectively).

653 Supplementary Fig. 4 Expression of mature amiRNAs in stably transfected cell lines. Total RNA

654 was isolated from BHK-21 cells stably transfected with the corresponding single or dual

655 amiRNA-expressing plasmids, and mature amiRNAs were quantitated by RT-stem loop real time

656 PCR as described by Chen et al (Chen, 2005). Mature amiRNA $290(A), \operatorname{amiRNA}_{444}(\mathrm{~B})$ and

657 amiRNA 1055 (C) expression from polycistronic vectors was normalized by glyceraldehyde-3-

658 phosphate deshydrogenase (GAPDH, A, B) or endogenous miRNA-706 (C) and relative

659 expression to the same amiRNA in mono-amiRNA polyclonal cells (horizontal line) was

660 calculated by the $2^{-\Delta \Delta C T}$ method. (D, E) Mature amiRNAs were quantitated in clonal cell lines

661 and relative expression to $\operatorname{amiRNA}_{290}$ (D) or $\operatorname{amiRNA}_{1055}$ (E) (horizontal lines) was calculated as 662 in (A).

663 
664 Supplementary Fig. 5. Partial 3D sequenced from RT-PCR amplified virus present in

665 supernatants of infected clonal amiRNA 290 (A) and amiRNA 1055 (B) cell lines at 48 hpi. Target

666 sequences are indicated by a box. 


\section{Figure 1}

RNAxs results of target prediction within FMDV 3D region.

Data are represented as the number of predicted target sequences (coverage) per 3D nucleotide. Total number of predicted sequences (gray areas) and sequences corresponding to the top 50 hits in the RNAxs ranking (dark gray areas) are shown. Target sequences selected for further study are indicated at the top of the corresponding peaks.

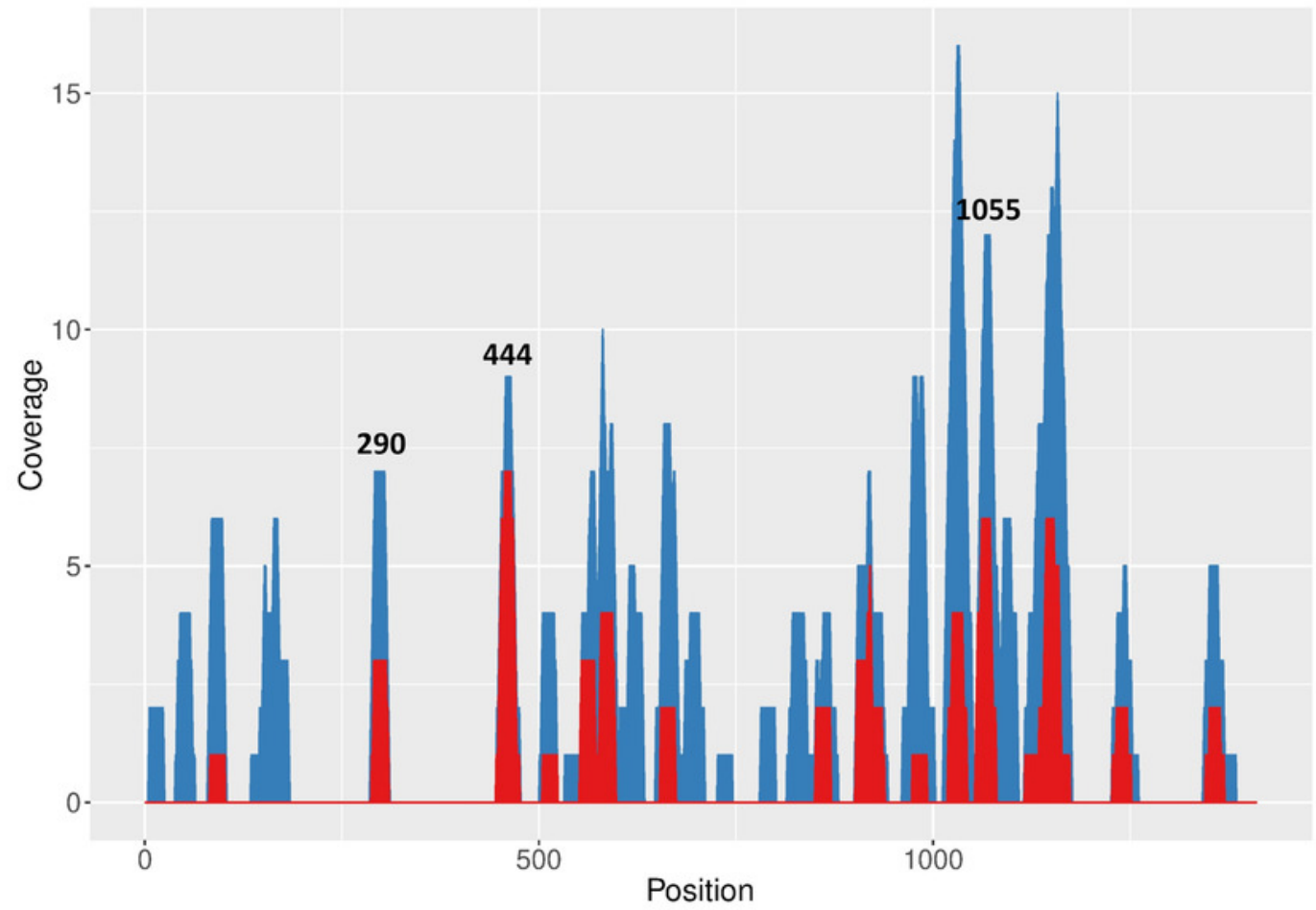


Figure 2

Silencing activity of small RNAs directed against FMDV 3D sequences.

Co-transfected cells were trypsinized and EGFP expression was analyzed by flow cytometry at $24 \mathrm{hpi}$ as described in Material and Methods. Cells were co-transfected with pEGFP3D and

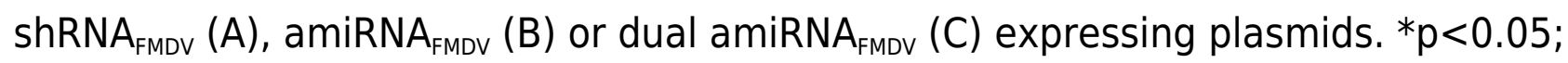
$* * p<0.01 ; * * * p<0.001$.

A
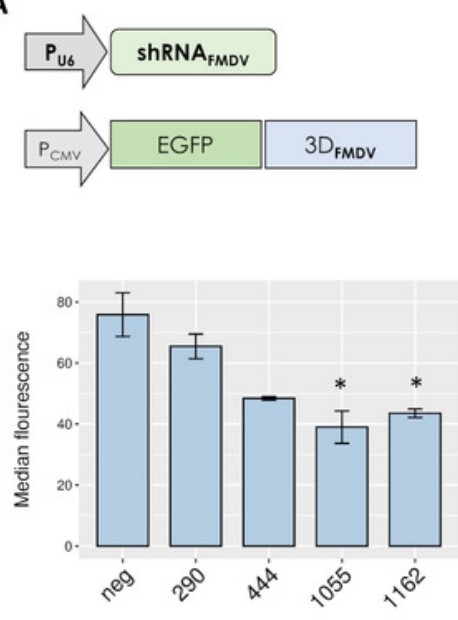

B
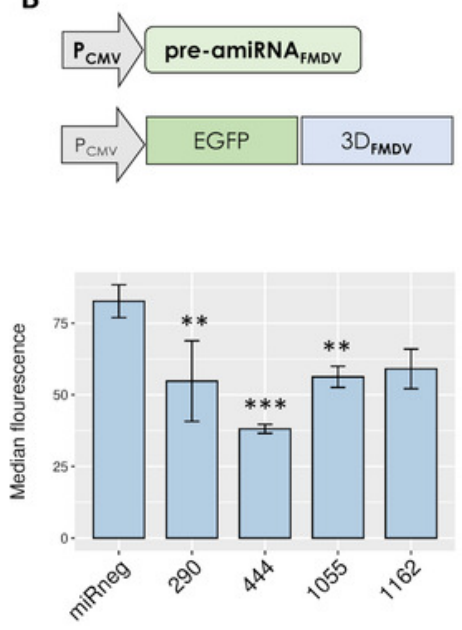

C
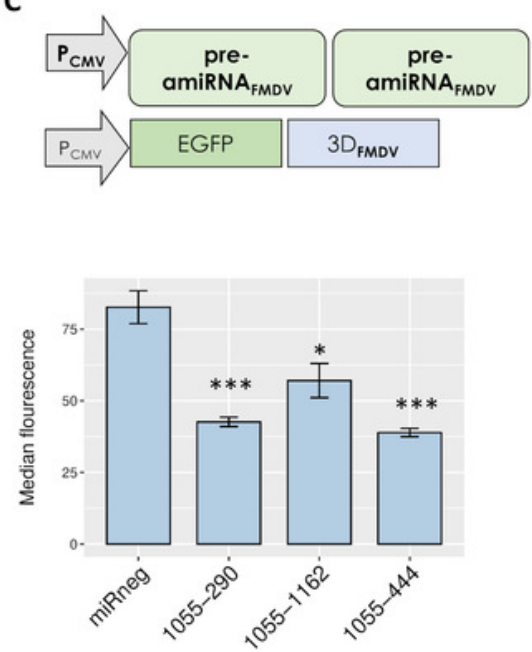


\section{Figure 3}

Antiviral effect of amiRNAs ${ }_{\mathrm{FMDV}}$ stably expressed in BHK-21 cells.

(A) Cytopathic effect observed in BHK-21 cell lines expressing amiRNA $\mathrm{FMDV}_{\text {and }}$ and control group (cell lines expressing miRneg) at $48 \mathrm{~h}$ post-infection. Cellular detachment, rounding, and destruction of the control group were more severe in the control group than in the experimental group (Magnification 100x). (B) Morphology and (C) dimensions of lysis plaques produced by FMDV in amiRNA FMDV -expressing cell lines and control cells. FMDV infection produced less plaques of smaller dimensions in cell lines expressing amiRNA than in control cells. ${ }^{* * *} p<0.0001$. (D-F) Viral titers in supernatants of infected cells were determined by the $\mathrm{TCID}_{50}$ method at $18 \mathrm{hpi}(\mathrm{D}), 24 \mathrm{hpi}(\mathrm{E})$ or $48 \mathrm{hpi}(\mathrm{F}) .{ }^{* *} \mathrm{p}<0.01$.
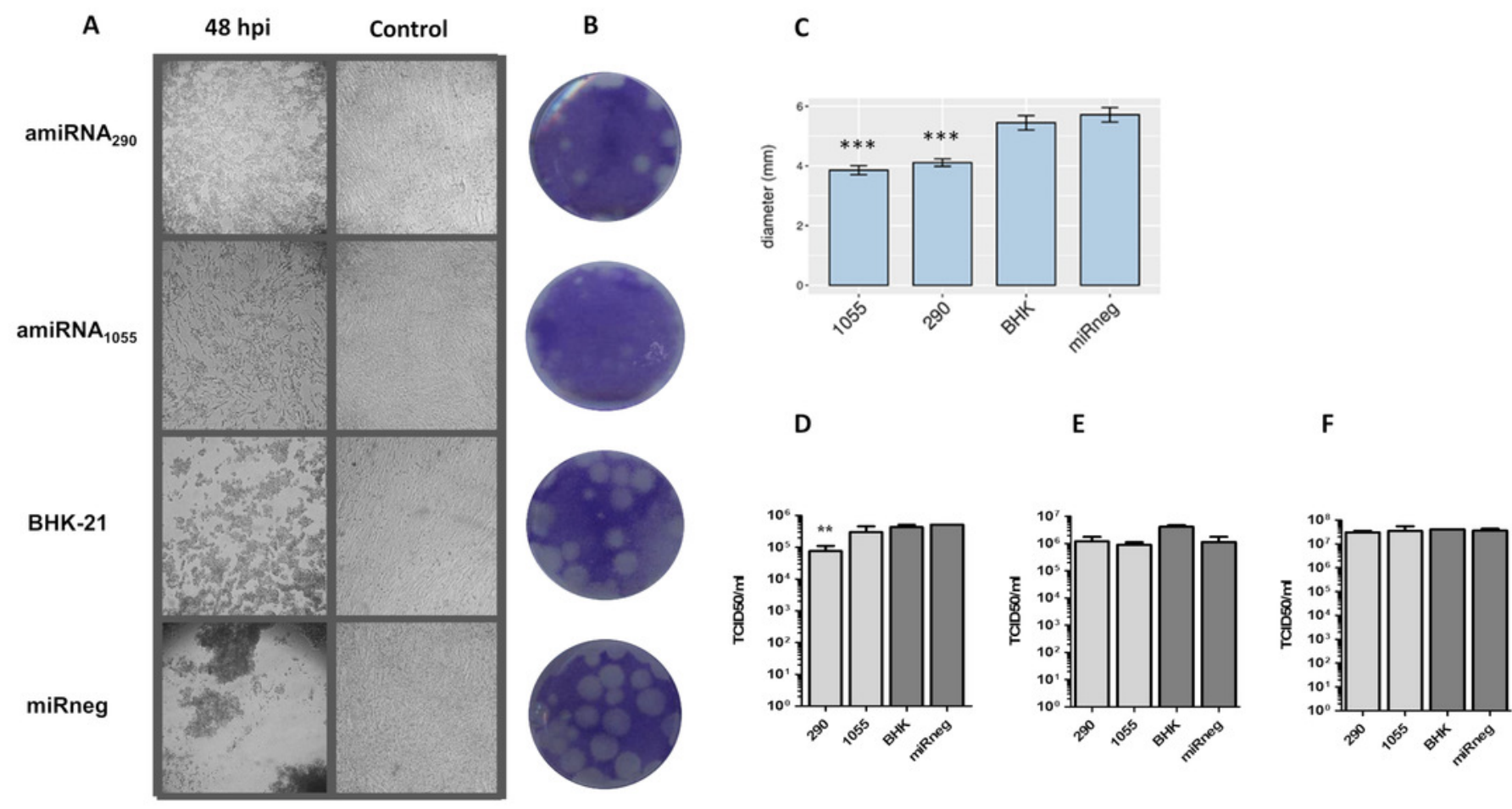
Figure 4

Antiviral activity of cloned amiRNA ${ }_{290}(\mathrm{~A}-\mathrm{C})$ and amiRNA ${ }_{1055}(\mathrm{D}-\mathrm{F})$ cell lines.

Cell lines were obtained from polyclonal cell lines by limiting dilution as described in Material and Methods. Cells were infected with FMDV A/Arg/01 at a low moi and viral titers in supernatants at $18 \mathrm{hpi}(\mathrm{A}, \mathrm{D}), 24 \mathrm{hpi}(\mathrm{B}, \mathrm{E})$ or $48 \mathrm{hpi}(\mathrm{C}, \mathrm{F})$ were determined by end-point dilution. ${ }^{*} p<0.05$ (as compared to miRneg cells, Student's $t$-test).

A

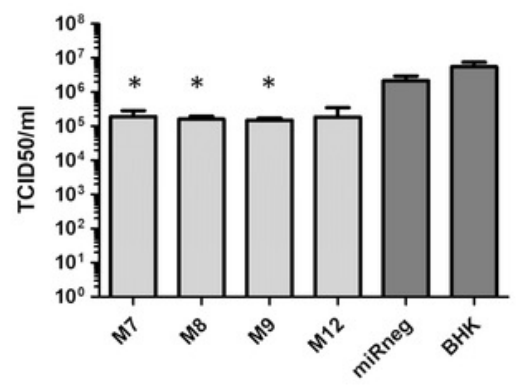

D

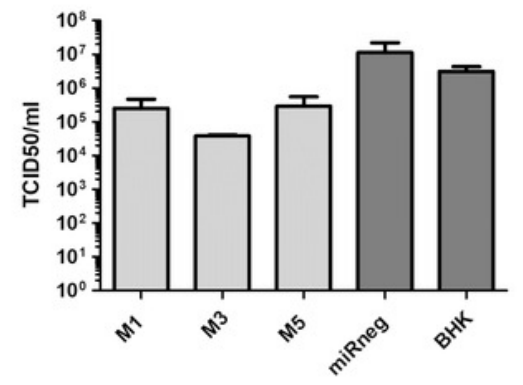

B

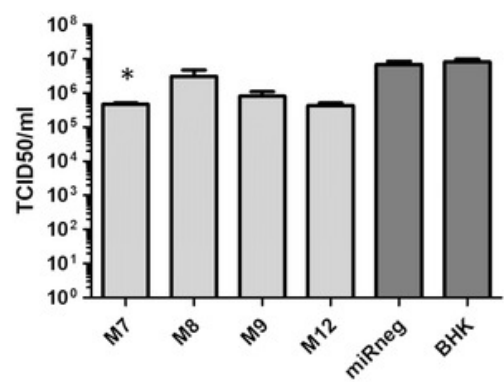

E

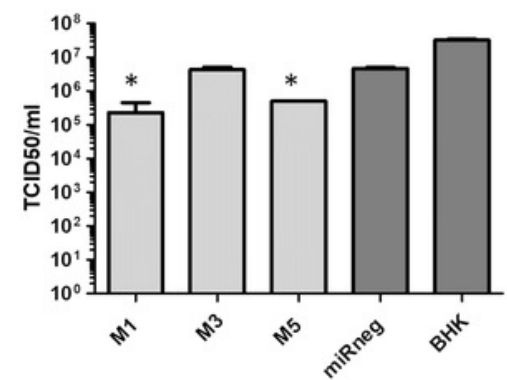

C

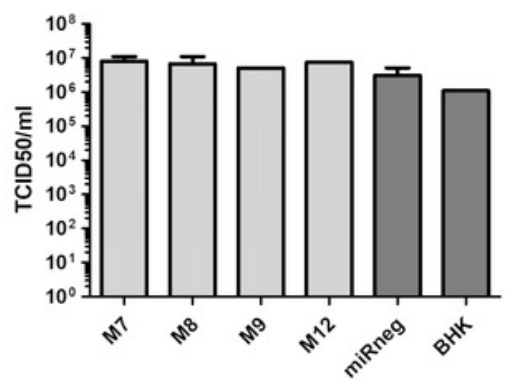

$\mathbf{F}$

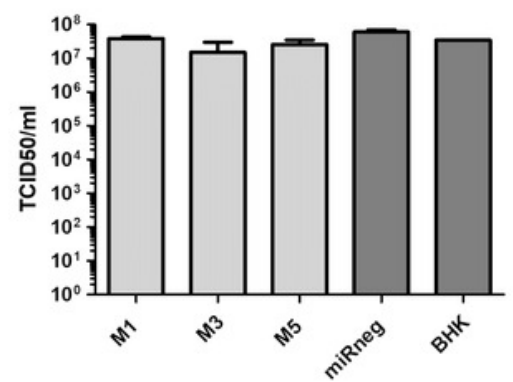




\section{Table $\mathbf{1}$ (on next page)}

Accessibility parameters of selected target sequences. 


\begin{tabular}{|c|c|c|c|c|c|}
\hline $\begin{array}{l}\text { Predicted target sequence (5'- } \\
\left.3^{\prime}\right)^{\mathrm{a}}\end{array}$ & $\begin{array}{c}\text { Mature sRNA sequence } \\
\left(5^{\prime}-3^{\prime}\right)^{\mathbf{b}}\end{array}$ & $\begin{array}{l}\text { RNAxs } \\
\text { ranking } \\
\text { position }^{\mathrm{c}}\end{array}$ & $\begin{array}{c}\text { Accessible } \\
\text { window } \\
\text { (starting } \\
\text { position; } \\
\text { length) } \\
\end{array}$ & $\begin{array}{c}\Delta \mathbf{G}_{\text {total }} \\
(\mathrm{Kcal} / \mathrm{mol})^{\mathrm{d}}\end{array}$ & $\begin{array}{c}\text { SRNA } \\
\text { ID }^{\mathrm{e}}\end{array}$ \\
\hline CGUUUACGAAGCAAUCAAA & CUUUGAUUGCUUCGUAAACGC & 2,3 & $13 ; 9$ & -22.2 & 290 \\
\hline GGAGAACAGAGAAUACAAA & UAUUCUCUGUUCUCCAUGAGC* & 1,6 & $17 ; 5$ & -22 & 444 \\
\hline \multirow[t]{3}{*}{ CUCAGGCCCCACUUUAAAU } & AUUUAAAGUGGGGCCUGAGAG & 5 & $11 ; 16$ & -29.6 & 1055 \\
\hline & AUAGUCCAUGUGGAAGUGUCU & & $18 ; 5$ & & 1162 \\
\hline & AAAUGUACUGCGCGUGGAGAC & & & & Neg \\
\hline
\end{tabular}

1 a 19 nt target sequences were predicted with RNAxs software.

2 b $21 \mathrm{nt}$ target sequences were defined according to the rules described in the Results section. The 2 nucleotides 3 incorporated to the $19 \mathrm{nt}$ predicted sequence are shown in bold.

$4{ }^{\mathrm{c}}$ Ranking position of $19 \mathrm{nt}$ predicted target sequences comprised within the $21 \mathrm{nt}$ predicted sRNA sequences.

$5{ }^{\mathrm{d}} \Delta \mathrm{G}_{\text {total }}$ was calculated with OligoWalk software, implemented in RNA structure package.

$6 \quad$ e sRNAs 1162 and Neg were used as positive and negative controls, respectively.

$7 *$ sRNA $_{444}$ slightly differed from the predicted target sequence to favor a less structured RNA conformation at the

8 target 3' end (Supplementary Figure 1) 


\section{Table 2 (on next page)}

Antiviral activity against FMDV exerted by amiRNAs and shRNAs at $5 \mathrm{hpi}$. 
1

2

\begin{tabular}{ccc}
\hline Target region & \multicolumn{2}{c}{ inhibition } \\
& amiRNA & shRNA \\
\hline 290 & 96 & 48 \\
444 & 90 & 87 \\
1055 & 71 & 77 \\
1162 & 60 & 10 \\
$1055-290$ & 90 & nd \\
$1055-444$ & 74 & nd \\
$1055-1162$ & 61 & nd
\end{tabular}

3

nd: not done

4

5

6 